ARTICLE

\title{
Non-fullerene acceptor organic photovoltaics with intrinsic operational lifetimes over 30 years
}

Yongxi Li (1) ${ }^{1}$, Xiaheng Huang ${ }^{1}$, Kan Ding ${ }^{1}$, Hafiz K. M. Sheriff, Jr (10 ${ }^{2}$, Long Ye ${ }^{3,4}$, Haoran Liu ${ }^{5}$, Chang-Zhi Li ${ }^{5}$,

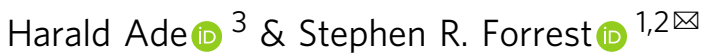

Organic photovoltaic cells (OPVs) have the potential of becoming a productive renewable energy technology if the requirements of low cost, high efficiency and prolonged lifetime are simultaneously fulfilled. So far, the remaining unfulfilled promise of this technology is its inadequate operational lifetime. Here, we demonstrate that the instability of NFA solar cells arises primarily from chemical changes at organic/inorganic interfaces bounding the bulk heterojunction active region. Encapsulated devices stabilized by additional protective buffer layers as well as the integration of a simple solution processed ultraviolet filtering layer, maintain $94 \%$ of their initial efficiency under simulated, 1 sun intensity, AM1.5 G irradiation for 1900 hours at $55^{\circ} \mathrm{C}$. Accelerated aging is also induced by exposure of light illumination intensities up to 27 suns, and operation temperatures as high as $65^{\circ} \mathrm{C}$. An extrapolated intrinsic lifetime of $>5.6 \times 10^{4} \mathrm{~h}$ is obtained, which is equivalent to 30 years outdoor exposure.

\footnotetext{
${ }^{1}$ Departments of Electrical Engineering, Material Science and Engineering, and Physics, University of Michigan, Ann Arbor, MI 48109, USA. ${ }^{2}$ Applied Physics Program, University of Michigan, Ann Arbor, MI 48109, USA. ${ }^{3}$ Department of Physics and Organic and Carbon Electronics Laboratories (ORaCEL), North Carolina State University, Raleigh, NC 27695, USA. ${ }^{4}$ School of Materials Science and Engineering and Tianjin Key Laboratory of Molecular Optoelectronic Sciences, Tianjin University, 300072 Tianjin, China. ${ }^{5}$ State Key Laboratory of Silicon Materials, MOE Key Laboratory of Macromolecular Synthesis and Functionalization, Department of Polymer Science and Engineering, Zhejiang University, 310027 Hangzhou, China. ${ }^{凶}$ email: stevefor@umich.edu
} 
O rganic photovoltaic cells (OPVs) have sparked considerable interest in recent years owing to their lightweight, flexibility, low cost, and environmental friendliness. As distinguished from incumbent solar technologies, OPVs have shown promise for use in building-integrated energy generating windows and greenhouses due to their high absorption in the near infrared while being semitransparent, and importantly, neutral optical density in the visible $e^{1-7}$. Recently, ladder-type non-fullerene acceptors (NFAs) have led to OPV power conversion efficiencies (PCEs) of $\sim 18 \%$ in opaque cells, and $10 \%$ in semitransparent cells with $50 \%$ visible transparency ${ }^{8-13}$. Although a few reports show that NFA-based solar cells under light-emitting diode (LED) illumination have the potential to reach long operational lifetimes (Supplementary Table 1), unfortunately, their ability to withstand use in harsh and realistic environments (significant ultraviolet (UV) infrared (IR), spectral content, and high operating temperatures) over long periods is, as yet, largely unproven ${ }^{14-20}$. In addition, high efficiency and long lifetime have yet to be simultaneously achieved in the same NFAbased cell. This has led to the belief that short operational lifetimes are an intrinsic disadvantage of high efficiency, NFA-based solar cells ${ }^{14,15}$

Indeed, a pervasive myth associated with OPVs is that the materials are intrinsically vulnerable to degradation and morphological instabilities in the bulk heterojunction $(\mathrm{BHJ})$ over the short term ${ }^{21-23}$. However, this myth is challenged by the very long extrapolated lifetimes (27,000 years) recently demonstrated in an archetype, thermally evaporated fullerene-based material system $^{24}$, providing a proof-of-concept that OPVs can have exceptional operational lifetimes. Compared to the highly stable devices employing a $\mathrm{C}_{70}$ acceptor with its high bond-dissociation energy, the most efficient NFAs contain multiple thiophene units with relatively weak chemical bonds that are dissociated at high UV and IR light intensities ${ }^{21-23}$. Therefore, it remains an open question as to what the degradation mechanisms are of solutionprocessed systems based on NFAs. Beyond changes in the active $\mathrm{BHJ}$ in both materials and morphology, the properties of organic/ electrode interfaces can also affect cell performance over time. Although strategies have been proposed to modify materials interfaces to suppress the degradation, to our knowledge, long term stable NFA-based solar cells under simulated air mass (AM) $1.5 \mathrm{G}$ irradiation have not yet been realized ${ }^{14-17}$. The causes of instability for these high performance NFA systems are also still not well understood. This motivates and encourages us to investigate the correlation between materials, film morphology, device architecture, and their relationship to the reliability of NFA OPVs.

In this work, we study solution-processed, archetype single junction solar cells with efficiency competitive with similar acceptor-donor-acceptor (A-D-A) type NFA-based solar cells. Importantly, previously our group showed that semitransparent OPVs based on the same materials systems studied here can achieve high performance and potentially low cost when used in power generating windows 25,26 . This leaves the remaining unfulfilled promise of long operational lifetime. The solar cells show only a $6 \%$ loss in initial efficiency under simulated AM1.5 G, one sun intensity irradiation after $1900 \mathrm{~h}$ exposure. To facilitate data comparison between laboratories and, consequently, the identification of various degradation factors and failure mechanisms, ISOS level L-2 protocols are followed. These protocols are used to evaluate the reliability of NFA solar cells, which tests their intrinsic stability under illumination and at elevated temperatures ${ }^{27}$. Additionally, the aging is accelerated by exposure of the cells to illumination intensities as high as 27 suns, and temperatures up to $65^{\circ} \mathrm{C}$. The degradation rate is found to increase superlinearly with intensity, but there is no systematic dependence on temperature, leading to an extrapolated intrinsic lifetime, corresponding to a decrease in PCE of $20 \%$ from its initial value, of $T_{80}>5.6 \times 10^{4} \mathrm{~h}$, which is equivalent to 30 years of outdoor operation.

\section{Results}

To investigate the reliability of NFA-based devices, we choose a material system used for semitransparent solar cell modules consisting of the commonly used donor, PCE-10, and an archetype near infrared (NIR) absorbing non-fullerene acceptor, BTCIC, whose molecular structures are shown in Fig. 1a (see Methods for the nomenclatures of all molecules used in this study). An inverted device was fabricated with the structure: (ITO) / ZnO (30 nm) / PCE-10:BT-CIC (1:1.5, w/w, $80 \mathrm{~nm}) /$ $\mathrm{MoO}_{\mathrm{x}}(10 \mathrm{~nm}) / \mathrm{Al}(100 \mathrm{~nm})$. To prevent chemical and morphological changes at organic/inorganic interfaces over time, buffer layers are inserted between the $\mathrm{BHJ}$ and charge transporting layers for improving the stability of the contact interface. As shown in Fig. 1a, several buffer materials are investigated for their influence on device reliability. The performance of the asgrown devices are listed in Supplementary Table 2 and 3 . Figure 1b-e and Supplementary Fig. 1-2 shows the evolution of the packaged solar cell performance parameters over time under simulated AM1.5 G, one sun intensity illumination by Xe-arc lamp solar simulator at $55 \pm 5^{\circ} \mathrm{C}$ for both control and buffercontained devices. All devices were encapsulated in a glovebox filled with ultrahigh purity nitrogen $\left(<0.1 \mathrm{ppm} \mathrm{O}_{2}\right.$ and $\left.\mathrm{H}_{2} \mathrm{O}\right)$ by bonding a glass cover slide to the substrate using a bead of UVcurable epoxy around the substrate periphery (see Methods). Aging in a light soaking chamber was performed on the encapsulated devices in air at a relative humidity of $\sim 50 \%$.

As an example of the influence of buffer layers, a self-assembled monolayer (IC-SAM) and a $2 \mathrm{~nm}$-thick $\mathrm{C}_{70}$ layer are applied at the electron and hole transporting layer interfaces with the $\mathrm{BHJ}$, respectively. One group of cells was continuously exposed to one sun intensity, simulated AM1.5G illumination at open circuit for 125 days, while the external quantum efficiencies (EQEs) and PCEs of the cells were periodically measured. A second, identical group of cells was aged at near the maximum power point (MPP). The control cell characteristics with no buffer layers decrease much faster, falling to less than $40 \%$ of their initial values within $2000 \mathrm{~h}$, with $T_{80}=60 \mathrm{~h}$, due to a rapid decrease in open-circuit voltage $\left(V_{O C}\right)$ and fill factor $(F F)$. However, the decrease in short circuit current $\left(J_{S C}\right)$ is relatively minor, consistent with results reported for most NFA solar cells ${ }^{16,27}$. When the IC-SAM is deposited between the $\mathrm{BHJ}$ and the $\mathrm{ZnO}$ layer, the change in $V_{O C}$ is suppressed, while $J_{S C}$ and $F F$ decrease slightly. The PCE retains $73 \%$ of its initial value after $2000 \mathrm{~h}$. On the other hand, with the incorporation of an anode buffer $\left(\mathrm{C}_{70}\right.$ or NPD) between $\mathrm{BHJ}$ and $\mathrm{MoO}_{\mathrm{x}}$, the degradation of $J_{S C}$ is reduced (Fig. 1d and Supplementary Fig. 2). The changes in $V_{O C}$ and $F F$, however, remain similar to that of the control. Devices with both cathode and anode buffers exhibits significantly improved stability, with PCE reduced by $<20 \%$ over 2000 h. Both $V_{O C}$ and $J_{S C}$ are stabilized with only a slight decrease in the FF. When a UV filter (with a wavelength cutoff at $400 \mathrm{~nm}$ ) is attached on the distal surface of the substrate, the efficiency is further stabilized, remaining at $92 \%$ of its initial value after $3000 \mathrm{~h}$. Notably, a similar result is observed between devices aged at open circuit and at MPP, as shown in Supplementary Fig. 3. The PCE retains $94 \%$ of its initial value after $2400 \mathrm{~h}$. The changes in $J_{S C}$ when measured under continuous illumination from a Xe-arc lamp are attributed to a redshift of the lamp spectrum with time ${ }^{24}$.

To evaluate the efficacy of increasing temperature and light intensity in accurately predicting the stability of the OPVs under standard reporting conditions (1 sun intensity, AM1.5 G 
(a)

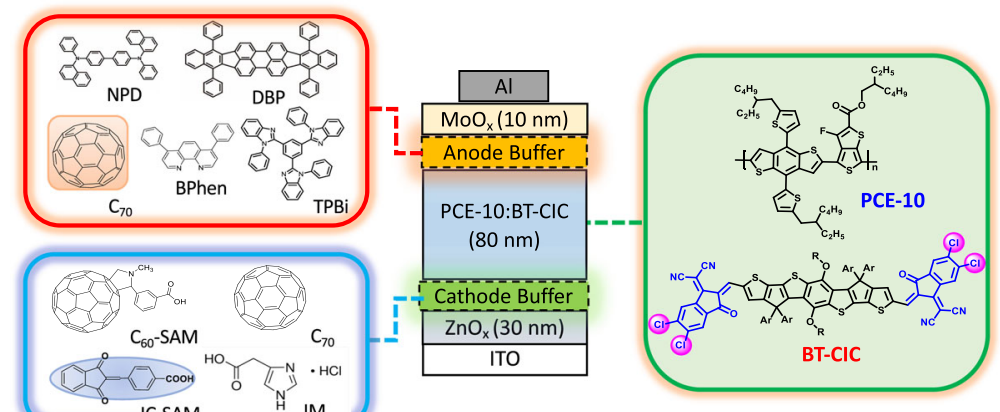

(b)

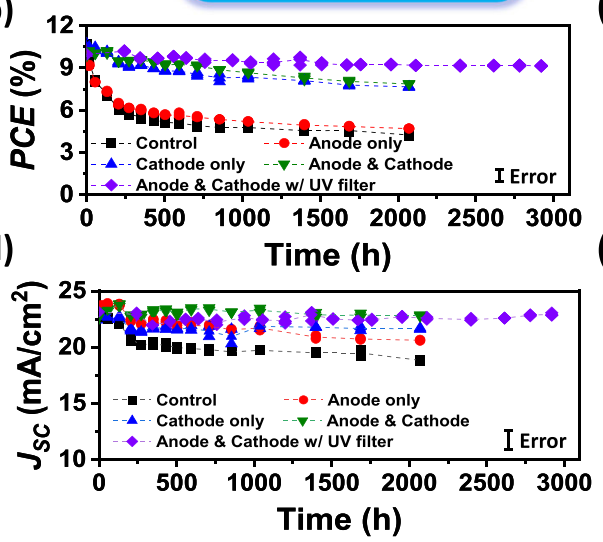

(c)

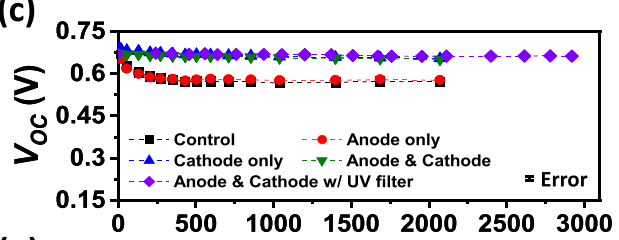

(e)

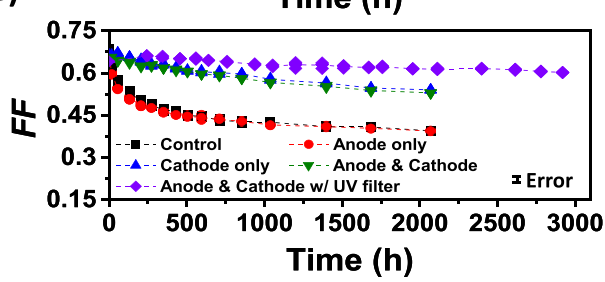

Fig. 1 Device structure, molecular structures, and OPV ageing data under 1 sun simulated AM1.5 G illumination. a Schematic of the device showing layer thicknesses and compositions (right): molecular structural formulae of the PCE-10 and BT-CIC (left): molecular structural formulae of the cathode and anode buffer materials. b PCE power conversion efficiency, $\mathbf{c} V_{O C}$ Open-circuit voltage, $\mathbf{d} J_{S C}$ Short circuit current, and e $F F$ Fill factor, plotted vs. aging time under 1 sun simulated AM1.5 G illumination for $3000 \mathrm{~h}$ with different device architectures (populations of 3-4 devices). The error bars indicate the 1 s.d. uncertainty of each measurement.

(a)

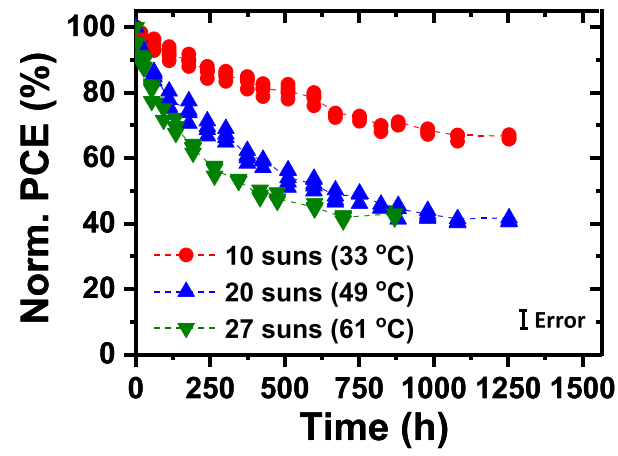

(b)

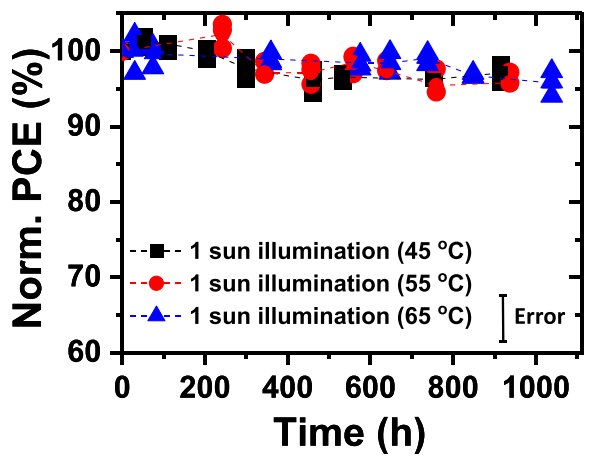

Fig. 2 OPV efficiency under accelerated aging conditions. a Normalized PCE plotted vs. aging time under illumination equivalent to $10 \pm 1.2,20 \pm 2.5$, and $27 \pm 3.8$ suns. b Normalized PCE plotted vs. aging time under simulated AM1.5 G one sun illumination with temperature of $45 \pm 5,55 \pm 5$, and $65 \pm 5{ }^{\circ} \mathrm{C}$ (populations of 3-4 devices). The error bars indicate the 1 s.d. uncertainty of PCE measurements.

spectrum, $\left.25^{\circ} \mathrm{C}\right)^{28}$, the cells are exposed to light intensity to up to 27 suns using white light-emitting diodes (LEDs, see Methods). Provided that the effects of temperature and intensity on device aging are independent, the degradation acceleration factor given by $^{28}$ :

$$
A=\left(\frac{I_{\text {test }}}{I_{\text {ref }}}\right)^{\gamma} \exp \left[-\frac{E_{a}}{k_{B}}\left(\frac{1}{T_{\text {test }}}-\frac{1}{T_{a}}\right)\right]
$$

allows for extrapolation to a reference intensity of $I_{r e f}=1$ sun when employing an elevated test intensity of $I_{\text {test }}$, and for determining the lifetime at ambient temperature, $T_{a}$, for a test done at $T_{\text {test }}$. Here, $E_{a}$ is the activation energy for failure, $k_{B}$ is Boltzmann's constant, and $\gamma$ is the intensity-dependent acceleration factor. Figure 2a and Supplementary Fig. 4 show the performance characteristics of a population of OPV cells with both IC-SAM and $C_{70}$ buffer layers aged at equivalent intensities of $10 \pm 1.2$, $20 \pm 2.5$, and $27 \pm 3.8$ suns. To avoid excessive heating at high intensity, the OPV cells were actively water-cooled to maintain the temperature at $33 \pm 5,49 \pm 5$, and $61 \pm 5^{\circ} \mathrm{C}$, respectively. We observe that $J_{S C}$ significantly decreases during aging, while $V_{O C}$ and FF remain relatively stable. On the other hand, a second group of OPV cells with the same structure was aged by elevating the cell temperature under simulated AM1.5 G, one sun intensity illumination (see Methods). The evolution of solar cell performance over time is shown in Fig. 2b. We observed that the PCEs of the cells are stable, with no temperature-dependent increase in degradation rate up to $65 \pm 5^{\circ} \mathrm{C}$. 
While there are several sources for changes of OPV performance over time, none is more fundamental than the degradation of the organic molecules comprising the layer structure itself. To determine the chemical stability of the BHJ constituents, we performed nuclear magnetic resonance (NMR) spectroscopy on BT-CIC before and after aging under AM1.5 G one sun illumination (see Methods and Supplementary Fig. 5). There is no change after aging for $60 \mathrm{~h}$, which suggests that the BT-CIC molecule itself is stable under light exposure. A similar stability was observed for PCE-10; its molecular weight remained unchanged and no fragments are observed in gel permeation chromatography spectra throughout the $50 \mathrm{~h}$ aging period (Supplementary Fig. 6). The photostability of PCE-10:BT-CIC blend film was investigated by UV-Vis spectroscopy (Supplementary Fig. 7). The shape and intensity of the exciton absorption spectrum shows almost no changes after aging for over $1500 \mathrm{~h}$ under AM1.5 G one sun illumination. This was also confirmed by Fourier-transform infrared spectroscopy as shown in Supplementary Fig. 8, where no difference was observed between the fresh and aged devices.

The morphological stability of the PCE-10:BT-CIC (1:1.5, w/w) film was further investigated using grazing incidence wide-angle X-ray scattering (GIWAXS), and carbon K-edge resonant soft $\mathrm{X}$-ray scattering (R-SoXS) as functions of aging under AM1.5 G, 1 sun intensity illumination. As shown in Fig. 3a, the GIWAXS diffraction profile of the PCE-10:BT-CIC blend film shows a (010) peak in the out-of-plane direction at $\sim 1.8 \AA^{-1}$, and a (100) diffraction peak in the in-plane direction at $0.30 \AA^{-1}$ characteristic of BT-CIC. No changes in coherence lengths were observed in the diffraction profiles after aging. Further, both fresh and aged PCE-10:BT-IC blends show the same multi-length-scale

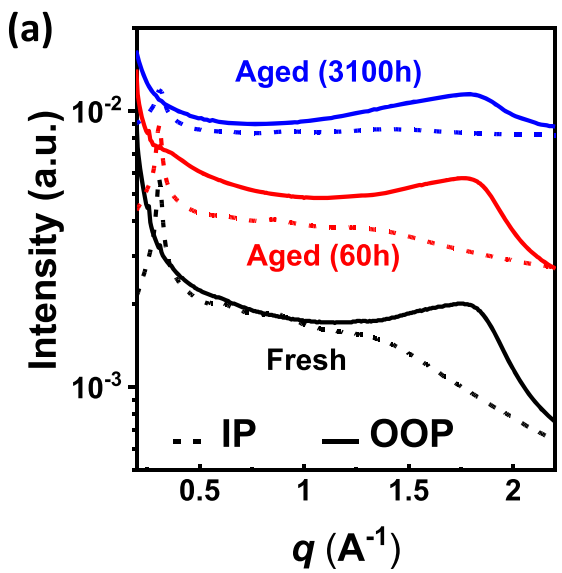

(c)

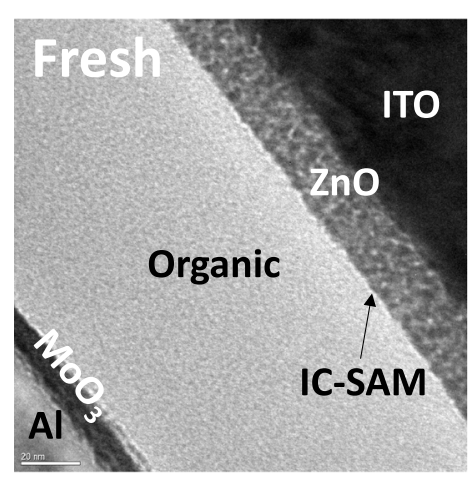

morphology with one peak at $q=0.10 \mathrm{~nm}^{-1}$ (corresponding to a distance of $63 \mathrm{~nm})$ and another at $0.28 \mathrm{~nm}^{-1}(22 \mathrm{~nm})$; see Supplementary Fig. 9, and a summary of the device performance over time in Table 1. Additionally, the composition of the BT-CIC molecule in the mixed domains was evaluated from the integrated scattering intensity (ISI) ${ }^{29}$, which scales with the standard deviation from the blend composition. This, in turn, is a function of the relative domain purity. The ISI remains constant over time, indicating that the bulk morphology of PCE-10:BT-CIC blend film is unchanged.

Transmission electron microscopy (TEM) images of thin crosssectional slices of the OPVs without, and with the IC-SAM interface buffer are shown in Fig. 3b-d, respectively. We find that the unprotected interface between $\mathrm{ZnO}$ and the $\mathrm{BHJ}$ appears broad, which is possibly due to the diffusion of $\mathrm{ZnO}$ into the organic layer. In contrast, when the IC-SAM is placed between the $\mathrm{ZnO}$ and the $\mathrm{BHJ}$, we observe a sharp boundary between layers that is stable over time.

The absorption loss of encapsulated PCE-10 and BT-CIC on ZnO films aged under high-intensity UV (365 nm LED, 60 suns, equivalent) is shown Fig. $4 \mathrm{a}-\mathrm{d}$. Contrary to previous studies where PCE-10 on ZnO showed no degradation over $1400 \mathrm{~h}$, UV soaking for $\sim 30 \mathrm{~h}$ bleaches the BT-CIC. This is attributed to the photo-oxidation of the exocyclic double bond in BT-CIC that interrupts the $\pi$-conjugation, thus decreasing its absorbance ${ }^{30,31}$. With insertion of the IC-SAM, no bleaching of BT-CIC and PCE10:BT-CIC blend films is observed, and the intramolecular charge transfer peak maintains both its shape and intensity (Fig. 4c, d and Supplementary Fig. 10). The X-ray photoelectron spectra (XPS) of the O $1 s$ core level are shown in Supplementary Fig. 11. We find the binding energy of oxygen at the $\mathrm{ZnO} / \mathrm{IC}-\mathrm{SAM}$

(b)

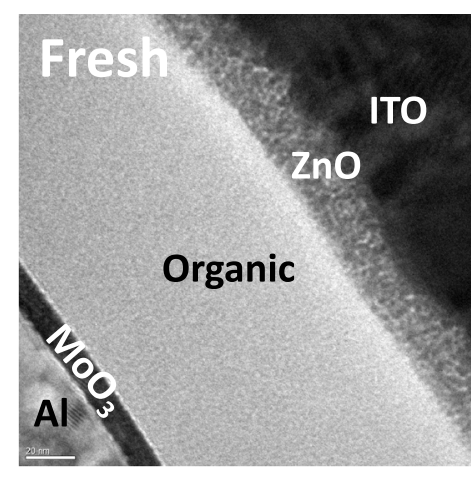

(d)

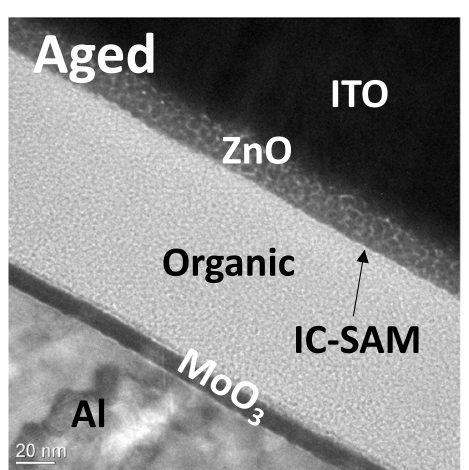

Fig. 3 GIWAXS characterization and TEM images. a In-plane (dotted line) and out-of-plane (solid line) sector-averaged profiles extracted from grazing incidence wide-angle X-ray scattering (GIWAXS) patterns; $q$ is the scattering vector. $\mathbf{b}$ Transmission electron microscope (TEM) image of cross-sectional slices of a fresh PCE-10:BT-CIC device without an interface buffer layer. c Fresh PCE-10:BT-CIC device with an IC-SAM layer inserted at the ZnO/BHJ interface. d Aged PCE-10:BT-CIC device with an IC-SAM layer inserted at the $\mathrm{ZnO} / \mathrm{BHJ}$ interface under $27 \pm 3.8$ suns illumination for $870 \mathrm{~h}$. 
Table 1 Morphological parameters extracted from GIWAXS and R-SoXS measurements.

\begin{tabular}{|c|c|c|c|c|c|}
\hline Fresh & $1.60 / 1.80$ & 0.30 & 0.10 & 63 & 0.69 \\
\hline Aged (60 h) & $1.60 / 1.80$ & 0.30 & 0.10 & 63 & 0.70 \\
\hline
\end{tabular}

(a)

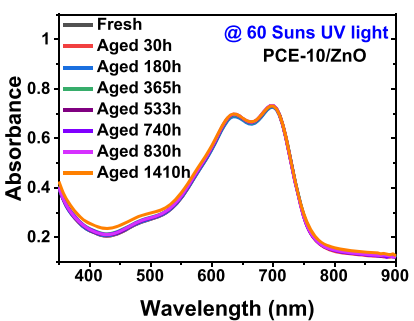

(e)

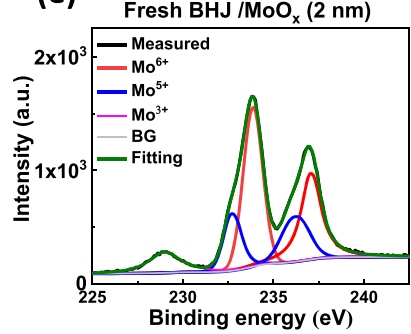

(b)

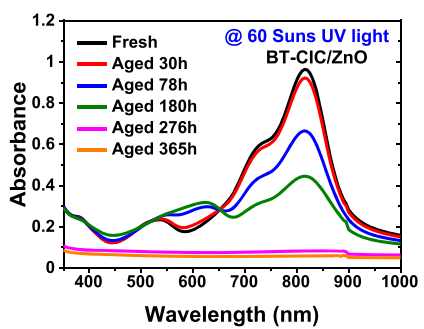

(f)

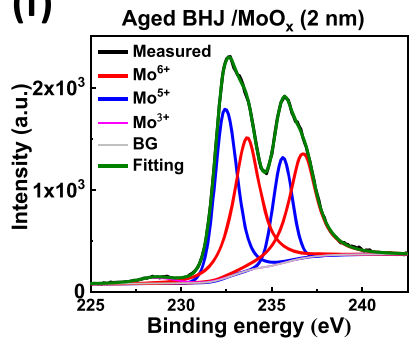

(c)

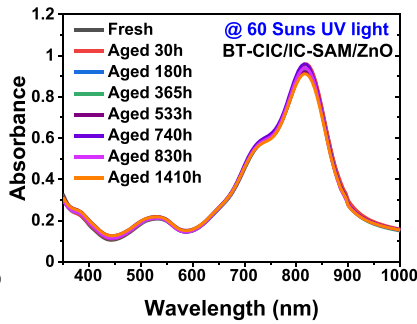

(g)

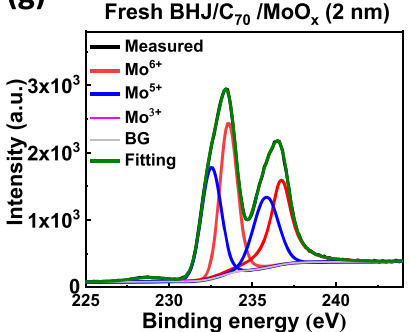

(d)

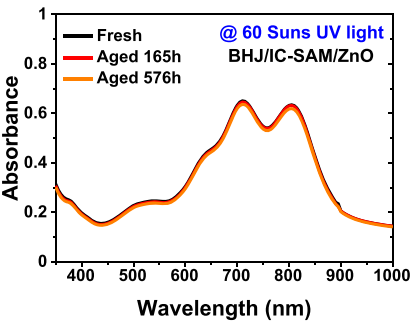

(h)

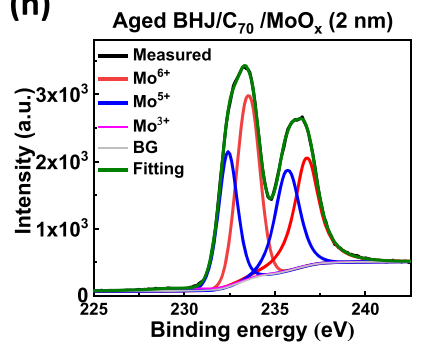

Fig. 4 Assessment of the stability of the organic/inorganic interface. UV-Vis absorption spectra plotted vs. aging time under ultraviolet illumination with intensity equivalent to 60 suns of thin film of a PCE-10 on $\mathrm{ZnO}, \mathbf{b} \mathrm{BT}-\mathrm{CIC}$ on $\mathrm{ZnO}, \mathbf{c} \mathrm{BT}-\mathrm{CIC}$ on $\mathrm{ZnO}$ with an IC-SAM buffer, and $\mathbf{d}$ PCE-10:BT-CIC on ZnO with an IC-SAM buffer. X-ray photoelectron spectra of Mo $3 d$ for the fresh device without e 2 nm $C_{70}$ layer, $\mathbf{f}$ aged device without $C_{70}$ layer, $\mathbf{g}$ fresh device with $C_{70}$ layer, and $\mathbf{h}$ aged device with $C_{70}$ layer. The Gaussian distributions used to fit the spectrum and the sum of these Gaussians are shown by the solid lines. The black lines, which are often buried by the summation fit (green lines), correspond to the experimental results.

interface is blue-shifted compared to samples with only $\mathrm{ZnO}$ or IC-SAM, suggesting that stabilizing chemical bonds are formed between $\mathrm{ZnO}$ and IC-SAM. This was further verified by the $\mathrm{C} 1 \mathrm{~s}$ core XPS spectra, where an ester group is identified at the $\mathrm{ZnO}$ / IC-SAM interface (Supplementary Fig. 12). Such surface passivation results in the reduced photoluminescent (PL) intensity of an oxygen interstitial trap state in $\mathrm{ZnO}$ (Supplementary Fig. 13) $)^{32}$.

One common source of instability of organic materials is their dissociation when exposed to high energy (i.e., UV) radiation ${ }^{22,33,34}$. Therefore, we used matrix-assisted laser desorption ionization with time-of-flight mass spectrometry (MALDI-TOF-MS) to investigate photostability of BT-CIC by identifying the chemical fragments generated during the UV aging. The MALDI-TOF-MS analysis shows two new chemical species that exhibit lower mass in comparison to BT-CIC, see Supplementary Fig. 14. The peak at a massto-charge ratio, $m / z=1596.3$, possibly originates from the scission of the $\mathrm{C}-\mathrm{O}$ bond out of the thiophene backbone. The electron donating character of the oxygen atom is known to weaken the $\mathrm{C}-\mathrm{H}$ bond, leading to the hydrogen abstraction on the $\mathrm{CH}_{2}$ group ( $\alpha$ position to the oxygen) ${ }^{22}$. On the other hand, the peak at $\mathrm{m} /$ $z=1433.1$ corresponds to a fragment with one peripheral phenyl group further removed. This result confirms that the weakest bonds in thiophene-based NFAs most susceptible to scission by UV irradiation are the side chain substituents.

\section{Discussion}

There are three-primary sources of degradation of PCE-10:BTCIC cells: photochemical reactions between the organic layers and the $\mathrm{ZnO}$ induced by exposure to $\mathrm{UV}$ illumination, increased concentration of defect states due to the penetration of Mo into the underlying organics (see Supplementary Fig. 15), and NFA bond dissociation when exposed to intense or prolonged UV radiation $^{35,36}$. As discussed above, a monolayer of IC-SAM stabilizes the $\mathrm{ZnO}$ surface, thereby preventing photochemical reactions with the organics. Similarly, an ultrathin $\mathrm{C}_{70}$ layer inserted between the organics and $\mathrm{MoO}_{\mathrm{x}}$ appears to substantially decrease anode- $\mathrm{BHJ}$ interactions. This is inferred from measurements of the Mo $3 d$ valence using X-ray photoelectron spectra (XPS) taken on PCE-10:BT-CIC $(1: 1.5, \mathrm{w} / \mathrm{w}, 80 \mathrm{~nm}) / \mathrm{MoO}_{\mathrm{x}}(2 \mathrm{~nm})$ and PCE10:BT-CIC $(1: 1.5, \mathrm{w} / \mathrm{w}, 80 \mathrm{~nm}) / \mathrm{C}_{70}(2 \mathrm{~nm}) / \mathrm{MoO}_{\mathrm{x}}(2 \mathrm{~nm})$ films before and after illumination for $250 \mathrm{~h}$ under simulated AM1.5 G, one sun intensity irradiation. As shown in Fig. $4 \mathrm{e}-\mathrm{h}$, the Mo $3 d$ spectra are fit by two $3 d$ doublets and a singlet, corresponding to Mo in three different oxidation states ${ }^{36-38}$. The peaks at $233.7 \mathrm{eV}$ and $236.7 \mathrm{eV}$ correspond to the $3 d$ doublet of $\mathrm{Mo}^{6+}$, those centered at $232.5 \mathrm{eV}$ and $235.6 \mathrm{eV}$ are the $3 d$ doublet of $\mathrm{Mo}^{5+}$, and the peak at $229.0 \mathrm{eV}$ is attributed to $\mathrm{Mo}^{3+}$. It is apparent that the peak intensity of $\mathrm{Mo}^{6+}$ at the $\mathrm{MoO}_{\mathrm{x}} / \mathrm{BHJ}$ interface decreases compared to the $\mathrm{MoO}_{\mathrm{x}} / 2 \mathrm{~nm} \quad \mathrm{C}_{70} / \mathrm{BHJ}$ structure after light soaking. This suggests that the insertion of only $2 \mathrm{~nm} \mathrm{C}_{70}$ between $\mathrm{BHJ}$ and $\mathrm{MoO}_{\mathrm{x}}$ forms an effective barrier that prevents reduction of the $\mathrm{Mo}^{6+}$ to $\mathrm{Mo}^{5+}$, possibly by preventing the interdiffusion of thiophene-rich BT-CIC into $\mathrm{MoO}_{\mathrm{x}}$. The conclusions drawn from these spectroscopic data are consistent with detailed analysis of the as-grown and aged $J-V$ characteristics of OPVs under 1 sun, simulated AM1.5 G illumination both with, and without the peripheral buffer layers found in the Supplementary Fig. 16, where the photogeneration efficiency of the BHJ decreases 
continuously in the control device with no buffer layers. In contrast, the value remains almost constant in the buffered devices, suggesting that the stability of bulk morphology essential to a high device reliability is improved by employing buffer layers and a UV filter ${ }^{39}$.

To test if the methodology demonstrated in this work could be applied to the other NFA solar cells, we also fabricated devices based on other two archetype NFAs: BT-IC and Y6 25,40 . The evolution of solar cell performance over time under simulated AM1.5 G one sun illumination is plotted in Supplementary Figs. 17 and 18. Similar to the devices with BT-CIC, the decrease in $J_{S C}, V_{O C}$, and FF are suppressed in PCE-10:BT-IC when ICSAM and $\mathrm{C}_{70}$ are inserted. Moreover, the degradation rate of OPV cells with buffer layers is at least 20 times less than in their absence without the elimination of UV effects. Additionally, the photostability of PM6:Y6 device was investigated ${ }^{9}$. The performance of device retains $90 \%$ of its initial value after aging for $500 \mathrm{~h}$ under AM1.5 G one sun illumination with a $400 \mathrm{~nm}$ long pass filter. This preliminary result indicates that NFA-based solar cells have the potential to meet the market needs of high reliability in addition to their high efficiency.

Since degradation depends on UV light exposure, we solution coat a $600 \mathrm{~nm}$-thick $\mathrm{ZnO}$ film on the distal surface of the glass substrate that effectively blocks radiation at wavelengths $<400 \mathrm{~nm}$ (Methods). Figure 5a shows the transmittance spectra of the fresh stand-alone filter, as well as one aged for $1500 \mathrm{~h}$ at 1 sun intensity in air. Compared to the bare glass substrate, the one comprising the $\mathrm{ZnO}$ filter exhibits negligible transmission at wavelengths $<375 \mathrm{~nm}$, with $\sim 6 \%$ Fresnel reflection loss at $>400 \mathrm{~nm}$. As a result, an OPV with the $\mathrm{ZnO}$ filter is significantly more stable (Fig. 5b-d). The performance of the integrated device under 1 sun intensity AM1.5 G illumination retained $94 \%$ of its initial PCE after $1900 \mathrm{~h}$ Supplementary Fig. 19). Additionally, the integrated device is stable under extremely high UV powers (60 suns equivalent UV). The performance of the filtered device retained $95 \%$ of its initial PCE after $340 \mathrm{~h}$, which is equivalent to 11 years of accumulated UV photons from outdoor exposure. In contrast, the PCE of the device lacking the UV filter declined by $66 \%$ over this same period.

The UV-filtered devices with both $\mathrm{C}_{70}$ and IC-SAM buffer layers were then subjected to accelerated aging to determine their stability extrapolated to standard reporting conditions. As shown in Fig. 6, the normalized PCE is plotted vs. the equivalent 1 sun exposure time at intensities ranging from 1 to 27 suns. We find that results at all intensities follow the same trend described by Eq. 1 (shown by the fit line). Given the OPV cells are thermally stable up to at least $65 \pm 5^{\circ} \mathrm{C}$, we can assume $E_{a} \approx 0$ under reasonable ranges of temperature around the standard reporting conditions. Furthermore, from the fit to all data in Fig. 6, we obtain $\gamma=2.02 \pm 0.16$. We speculate that the quadradically dependent degradation of the PCE-10:BT-CIC BHJ cells results from second-order reactions such as exciton-exciton and exciton-polaron annihilation ${ }^{41}$. On the basis of this value of $\gamma$, we can define an equivalent 1 sun exposure time for OPV cells aged at each intensity. Extrapolating these data, we obtain a $T_{80}>5.6 \times 10^{4} \mathrm{~h}$. To convert the lifetimes of the OPV cells into outdoor lifetime projections, we assume an average of $5 \mathrm{~kW}-\mathrm{h} / \mathrm{m}^{2}$ of sunlight per day ${ }^{42}$. In this case, $T_{80}$ extrapolates to $>30 \mathrm{yr}$ outdoor exposure, suggesting that the intrinsic stability of PCE10:BT-CIC cells can substantially exceed the requirements for almost all practical applications.

This work demonstrates that NFA-based solar cells have the ability to achieve long term intrinsic stability under circumstances that use our innovative buffering and filtering schemes. However, to be practical, the cost and stability of encapsulation must also be considered. Although recent breakthroughs in deposition technology, such as atomic layer deposition (ALD), have led to dramatic improvements in the barrier to gas penetration, and hence (a)

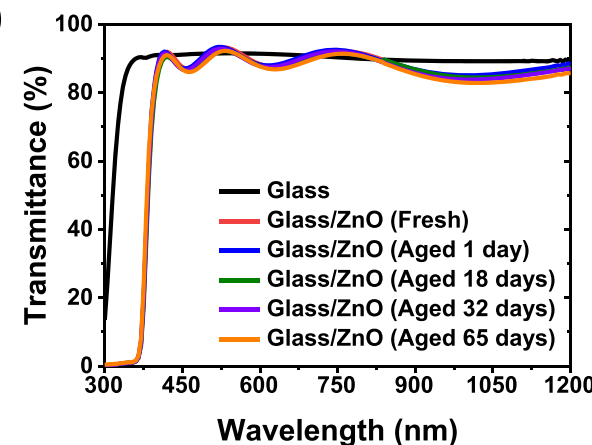

(c)

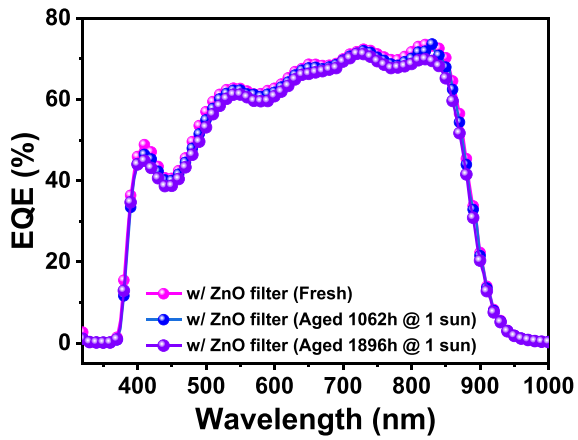

(b)

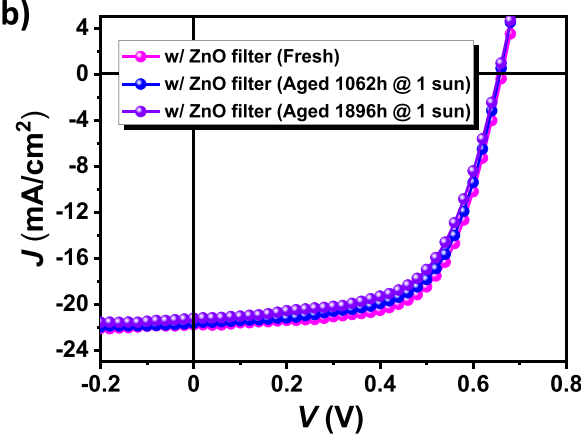

(d)

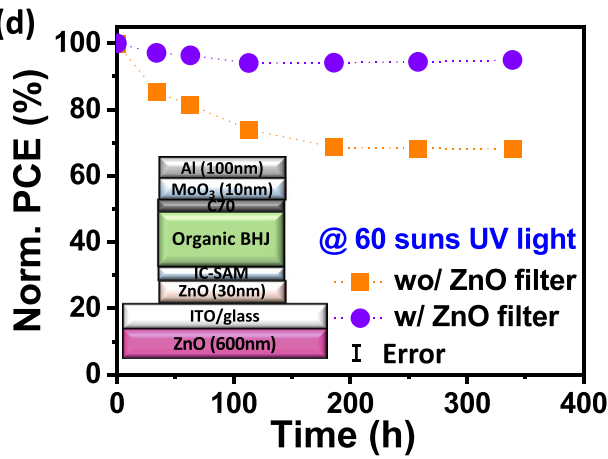

Fig. 5 Characterization of the ZnO UV filter. a UV-Vis absorption spectra of a ZnO UV filter plotted vs. aging time under simulated AM1.5 G 1 sun illumination in air over 65 days. b Current-density-voltage characteristics, and c external quantum efficiency (EQE) spectra of fresh PCE-10:BT-CIC (1:1.5, w/w) devices with a ZnO UV filter, as well as the aged device under simulated AM1.5 G 1 sun illumination. d Normalized PCE plotted vs. aging time under ultraviolet illumination equivalent to 60 suns for more than $350 \mathrm{~h}$ with and without $\mathrm{ZnO}$ UV filter. The error bars indicate the $1 \mathrm{~s} . \mathrm{d}$. uncertainty of PCE measurements. 


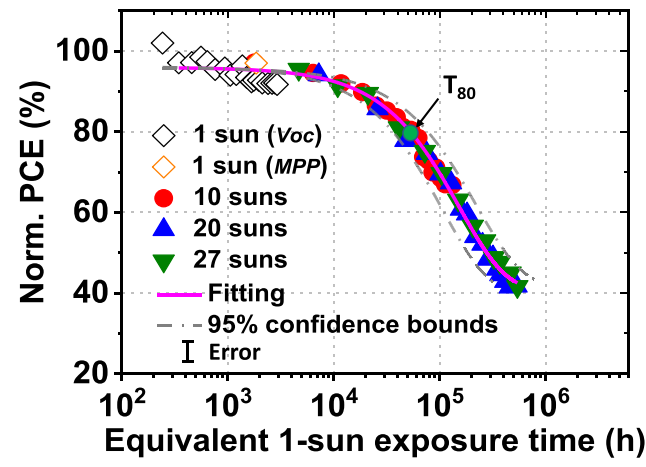

Fig. 6 Aging acceleration factor and extrapolated OPV lifetime.

Normalized PCE plotted vs. the equivalent 1 sun exposure time, defined as time (h) multiplied by intensity (suns) raised to the power of 2.02, for OPV cells under all illumination conditions used in this work. The data are fit to an exponential to estimate the time for the PCE to drop to $80 \%$ of its initial value, $T_{80}$, with the best fit shown as a solid line. The error bar indicates the 1 s.d. uncertainty of the PCE measurements.

improved reliability of organic light-emitting diodes (OLEDs), such layer growth is slow, and hence ultimately may be prohibitively costly ${ }^{28}$. Furthermore, barrier-coated plastic substrates are currently more costly to produce than metal foils or ultrathin glass substrates, and they also have compelling advantages such as toughness that may ultimately be more important than cost alone $^{28}$. For transparent OPVs, however, the cost of encapsulation can be minimized by inserting the thin-film modules into the pocket of double-pane windows sealed with inert gas, as is common practice for insulating windows. The costs for such systems have been estimated to be quite attractive ${ }^{26}$.

In summary, we demonstrate a solution-processed non-fullerene, thiophene-based acceptor OPV with requirements of high efficiency, low cost, and remarkable operational stability simultaneously achieved. The instability of the NFA solar cells originates from time-dependent changes at the interfaces between the $\mathrm{BHJ}$ and the inorganic charge transporting layers. The changes are significantly suppressed by insertion of the ultrathin protective buffer layers, IC-SAM and $\mathrm{C}_{70}$, at each side of the $\mathrm{BHJ}$. Further enhancement is afforded by the integration of a simple $\mathrm{ZnO}$ UV-filter layer to the distal surface of the glass substrate. This results in the PCE-10:BT-CIC solar cell that maintains 94\% of its initial efficiency under simulated, 1 sun intensity, AM1.5 G irradiation for $1900 \mathrm{~h}$ at $55^{\circ} \mathrm{C}$. An extrapolated lifetime of $T_{80}>5.6 \times 10^{4} \mathrm{~h}$ is obtained by exposure of light illumination intensities up to 27 suns, and operation temperatures as high as $65^{\circ} \mathrm{C}$. Our results show that NFA-based solar cells have the potential to meet the market needs of high reliability in addition to their high efficiency and potentially low manufacturing costs.

\footnotetext{
Methods

Materials. The non-fullerene acceptors are (4,4,10,10-tetrakis(4-hexylphenyl)-5,11(2-ethylhexyloxy)-4,10-dihydro-dithienyl[1,2-b:4,5b'] benzodithiophene-2,8-diyl) bis(2-(3-oxo-2,3-dihydroinden-5,6-dichloro-1-ylidene)malononitrile, BT-CIC, and (4,4,10,10-tetrakis(4-hexylphenyl)-5,11-(2-ethylhexyloxy)-4,10-dihydro-dithienyl[1,2 b:4,5b']benzodithiophene-2,8-diyl)bis(2-(3-oxo-2,3-dihydroinden-1-ylidene)malononitrile), BT-IC. The cathode buffer material is 4-((1,3-dioxo-1,3-dihydro- $2 \mathrm{H}$ inden-2-ylidene)methyl)benzoic acid, IC-SAM ${ }^{25,38,43,44}$. Other materials purchased from commercial suppliers are (4,4,10,10-tetrakis(4-hexylphenyl)-5,11-(2-ethylhexyloxy)-4,10-dihydro-dithienyl[1,2-b:4,5 $\left.\mathrm{b}^{\prime}\right]$ benzodithiophene-2,8-diyl)bis(2-(3-oxo2,3-dihydroinden-5,6-dichloro-1-ylidene) malononitrile, PCE-10, Poly[(2,6-(4,8bis(5-(2-ethylhexyl-3-fluoro)thiophen-2-yl)-benzo[1,2-b:4,5-b']dithiophene))-alt-(5,5(1',3'-di-2-thienyl-5',7'-bis(2-ethylhexyl)benzo[1',2'-c:4',5'-c']dithiophene-4,8-dione)], PM6, 2,2'-((2Z,2'Z)-((12,13-bis(2-ethylhexyl)-3,9-diundecyl-12,13-dihydro[1,2,5] thiadiazolo[3,4e] thieno[2”, $\left.3^{\prime \prime}: 4^{\prime}, 5^{\prime}\right]$ thieno [2',3': 4,5] pyrrolo[3,2-g]thieno[2',3':4,5] thieno[3,2-b]indole-2,10diyl)bis(methanylylidene))bis(5,6-difluoro-3-oxo-2,3-dihydro-1H-indene-2,1 diylidene) dimalononitrile, Y6 and 4-(1',5'-Dihydro- $1^{\prime}$-methyl-2'
}

$H$-[5,6]fullereno- $\mathrm{C}_{60}-\mathrm{I}_{\mathrm{h}}-[1,9-\mathrm{c}]$ pyrrol-2'-yl)benzoic acid, $\mathrm{C}_{60}-\mathrm{SAM}$ (1-Material, Dorval, CA), $\mathrm{C}_{70}$ SES Research, Houston, TX, US), 4-imidazoleacetic acid hydrochloride (Sigma-Aldrich, St. Louis, MO, US), N,N'-Di(1-naphthyl)-N,N'-diphenyl-(1,1'biphenyl)-4,4'-diamine, NPD, 5,10,15,20-Tetraphenylbisbenz[5,6]indeno[1,2,3cd: $1^{\prime}, 2^{\prime}, 3^{\prime}$-lm] perylene, DBP, and 2,2',2"-(1,3,5-Benzinetriyl)-tris(1-phenyl-1-H-benzimidazole), TPBi, Bathophenanthroline, Bphen from Luminescence Technology Corp., New Taipei City, Taiwan, $\mathrm{MoO}_{3}$ (Acros Organics, Fair Lawn, NJ, US), Zinc acetate dihydrate (Sigma-Aldrich, St. Louis, US) and Al (Alfa Aesar, Haverhill, MA, US)

Solar cell fabrication. Prepatterned ITO-coated glass substrates with sheet resistances of $15 \Omega /$ sq were purchased from Luminescence Technology Corp. Prior to thin-film deposition, the substrate surface was detergent and solvent cleaned with acetone and isopropanol, followed by $\mathrm{CO}_{2}$ snow cleaning and exposure to ultraviolet-ozone for $15 \mathrm{~min}^{45}$. The $\mathrm{ZnO}$ layer (ca. $30 \mathrm{~nm}$ ) was spin cast from a $\mathrm{ZnO}$ precursor solution onto the substrates and then thermally annealed at $150^{\circ} \mathrm{C}$ for $30 \mathrm{~min}$ in air. The IC-SAM was dissolved in methanol with a concentration of $1 \mathrm{mg} / \mathrm{ml}$ and spin coated at $4000 \mathrm{rpm}$ for $60 \mathrm{~s}$, followed by thermal annealing at $110^{\circ} \mathrm{C}$ for $10 \mathrm{~min}$. Then, the IC-SAM coated substrate was washed with methano to remove the residues. The non-fullerene active layer, PCE-10:BT-CIC (1:1.5 by weight), was dissolved in chlorobenzene:chloroform (CB:CF, 10:1 by vol.) with a concentration of $16 \mathrm{mg} / \mathrm{ml}$. The solution was filtered once with a $0.45 \mu \mathrm{m}$ polytetrafluoroethylene (PTFE) syringe filter prior to use, and then spin-coated onto the substrate at $2000 \mathrm{rpm}$ for $90 \mathrm{~s}$ to achieve a thickness of $80-90 \mathrm{~nm}$. The samples were transferred into the vacuum chamber connected to glove boxes filled with ultrapure $\mathrm{N}_{2}\left(\mathrm{O}_{2}, \mathrm{H}_{2} \mathrm{O}<0.1 \mathrm{ppm}\right)$. The $\mathrm{C}_{70}, \mathrm{MoO}_{3}$ and $\mathrm{Al}$ films were deposited at $0.2 \AA / \mathrm{s}$ in a high vacuum chamber with a base pressure of $10^{-7}$ torr. The deposition rates and thicknesses were measured using quartz crystal monitors and calibrated post-growth by variable-angle spectroscopic ellipsometry. Device areas of $2 \times 2 \mathrm{~mm}$ were defined by the overlap of the ITO anode and the Al cathode with an ultrathin shadow mask $(50 \mu \mathrm{m})$.

The ZnO UV filter was deposited on the distal end of the glass substrate before the device fabrication. The $\mathrm{ZnO}$ layer $(\mathrm{ca} .600 \mathrm{~nm})$ was spun cast from a $\mathrm{ZnO}$ precursor solution and followed by thermal annealing at $300{ }^{\circ} \mathrm{C}$ for $1 \mathrm{~h}$ in air. After cooling to the room temperature, the $\mathrm{ZnO}$-coated glasses were cleaned using a series of the solvents, acetone, and isopropanol, and sonicated 5 mins for each solvent.

Solar cell characterization. The current-density-voltage $(J-V)$ characteristics and external quantum efficiencies (EQE) of the cells were measured in a glovebox filled with ultrapure $\mathrm{N}_{2}$. The EQE measurements were performed with devices underfilled by a $200 \mathrm{~Hz}$-chopped monochromated and focused beam from a Xe lamp. The current output from the devices as well as from a reference National Institute of Standards and Technology (NIST)-traceable Si detector were recorded using a lockin amplifier. Light from a Xe lamp filtered to achieve a simulated AM1.5 G spectrum (ASTM G173-03) was used as the source for $J-V$ measurements. The lamp intensity is varied using neutral-density filters and was calibrated by a Si reference cell certified by National Renewable Energy Laboratory. Each cell was measured under six different light intensities from 0.001 sun to 1 sun $\left(100 \mathrm{~mW} / \mathrm{cm}^{2}\right)$. The $J_{S C}$ were calculated from the EQE spectrum, with $<7 \%$ relative mismatch of the measured $J_{S C}$ from $J-V$ characteristics. The error bars quoted in the tables take into account both the random and systematic errors.

Device stability measurements. All devices were encapsulated by bonding a glass cover slide to the substrate in a glovebox filled with high purity nitrogen, using an ultraviolet-curable epoxy. All aging in the light soaking chamber were performed on the encapsulated devices in air. The Xe-arc lamp spectrum (see Supplementary Fig. 20) was filtered to approximate an AM1.5 G reference, and its intensity was controlled to provide an output of $1.0 \pm 0.1 \mathrm{~kW} \mathrm{~m}^{-2}$ during the aging period. One group of packaged devices was aged under open circuit at $55 \pm 5^{\circ} \mathrm{C}$, and the photovoltaic efficiency was periodically recorded under AM1.5 G simulated spectrum. A second group of encapsulated devices was mounted on printed circuit boards and connected to a resistor to fix its current and voltage near the maximum power operating point. The PCE was recorded every $2 \mathrm{~h}$. For the UV degradation mechanism studies, a $400 \mathrm{~nm}$ cutoff UV filter (Thorlabs) was placed between the $\mathrm{OPV}$ and the illumination source. Thermal stability tests were performed by aging the devices at open circuit between $45 \pm 5^{\circ} \mathrm{C}$ and $65 \pm 5^{\circ} \mathrm{C}$ in the light soaking chamber under 1 sun illumination. A resistive heater was placed on a $\mathrm{Cu}$ plate beneath each device to independently control temperature, which was monitored using a thermocouple. The photovoltaic efficiency was periodically recorded under AM1.5 G simulated spectrum.

High-intensity illumination from LEDs operating at various intensities was concentrated onto the OPVs with Ag-coated reflective light pipes. The LED intensity that produced a photocurrent equivalent to $J_{S C}$ under AM1.5 G illumination $\left(J_{S C}, \mathrm{AM} 1.5 \mathrm{G}\right.$ ) was equivalent to 1 sun intensity. To calibrate higher LED intensities, a neutral-density filter was used to ensure that the OPVs remained in their linear regime ${ }^{24,46}$. The neutral-density filter was $10 \%$ transmissive, thus the equivalent solar intensity (in units of suns) of the LEDs was calculated using the 
following equation:

$$
\text { Equivalent solar intensity }=\frac{10 J_{\text {photon, } L E D}}{J_{S C, A M 1.5 G}}
$$

To avoid excessive heating at high intensity, the OPV cells were actively watercooled with a closed-loop chiller to $33 \pm 5,49 \pm 5$, and $61 \pm 5^{\circ} \mathrm{C}$ at $10 \pm 1.2,20 \pm 2.5$, and $27 \pm 3.8$ suns, respectively. The ultraviolet-emitting LED intensity was measured using a calibrated Si photodiode and converted to a solar-equivalent intensity by dividing its output power by the power contained in the AM1.5 G spectrum at wavelengths $<400 \mathrm{~nm}\left(4.6 \mathrm{~W} \mathrm{~m}^{-2}\right)$. The responsivity of the Si photodiode was greater than $0.1 \mathrm{~A} \mathrm{~W}^{-1}$ over the wavelengths emitted by the ultraviolet LED. The spectra of the white LEDs and UV LED are shown in Supplementary Fig. 21.

Molecular structure characterization. The ${ }^{1} \mathrm{H}$ NMR spectra were collected using a Varian MR400 spectrometer in deuterated chloroform solution with trimethylsilane (TMS) as reference. Gel permeation chromatography (GPC) spectra were collected on a Shimadzu GPC system with THF as the solvent. The samples used for NMR and GPC measurements were fabricated on quartz and encapsulated with a cover glass similar to the OPV device. The package lids were removed after light soaking under 1 sun illumination. Laser desorption/ionization time-of-flight mass spectra were collected using a Bruker AutoFlex Speed MALDI-TOF instrument from the aged thin-film sample under nitrogen laser pulse illumination.

\section{Grazing incidence wide-angle X-ray scattering and resonant soft X-ray} scattering measurements. Samples were prepared on $\mathrm{Si}$ wafers in a similar manner to the OPV devices. The thin films were measured at beamline 7.3.3 at the Advanced Light Source (ALS) ${ }^{47}$, Lawrence Berkeley National Lab (LBNL). The X-ray energy was $10 \mathrm{keV}$ and the scattering patterns were recorded on a $2 \mathrm{D}$ image plate (Pilatus $1 \mathrm{M}$ ) with a pixel size of $172 \mu \mathrm{m}(981 \times 1043$ pixels). The samples were $\sim 10 \mathrm{~mm}$ long in the direction of the beam path, and the detector was located at a distance of $300 \mathrm{~mm}$ from the sample center (distance calibrated by a AgB reference). The incidence angle was $0.16^{\circ}$. Resonant soft X-ray scattering with photon energy of $283.6 \mathrm{eV}$ was performed at beamline 11.0.1.2 of LBNL ${ }^{48}$. Thin films were transferred onto a $\mathrm{Si}_{3} \mathrm{~N}_{4}$ substrate and the experiment was done in the transition mode.

Transmission electron microscopy (TEM). The cross-sectional slice of the OPV device was prepared by focused ion beam (FIB) milling (FEI Nova 200 Nanolab SEM/FIB) and glued by Pt soldering onto a FIB lift-out grid rod. The sample was then transferred into a TEM chamber (JEOL 2010F) to take images.

Thin-film absorption and transmission measurements. The absorbance and transmittance of thin films were collected using a calibrated PerkinElmer Lambda 1050 ultraviolet-visible spectrometer.

X-ray photoelectron measurements (XPS). XPS spectra were collected using a Kratos Axis Ultra XPS at the Michigan Center for Materials Characterization, with a monochromatic $\mathrm{Al}$ source $(10 \mathrm{~mA}, 14 \mathrm{kV})$, a pass energy of $20 \mathrm{eV}$ and step size of $0.1 \mathrm{eV}$ on a spot size of $700 \times 300 \mu \mathrm{m}$. The XPS curves were analyzed with CasaXPS software. To account for charge compensation, the $\mathrm{C} 1 \mathrm{~s}$ peak at $284.5 \mathrm{eV}$ was used to calibrate the energies. Each peak was analyzed using Gaussian-Lorentzian curves with a full-width half maxima less than two and a linear background.

Photoluminescence measurements. The PL spectra were measured using a $325 \mathrm{~nm}$ $\mathrm{He}-\mathrm{Cd}$ continuous wave laser with a pump power $\sim 50 \mu \mathrm{W}$. A notch filter at $325 \pm 5 \mathrm{~nm}$ was used to filter the background emission from the laser, and an additional $400 \mathrm{~nm}$ long pass filter is used to filter the pump laser at the end of the signal path. The output signal was coupled to the CCD via an optical fiber and monochromator. All samples used for PL measurement were prepared on the quartz substrates.

\section{Data availability}

The data supporting the results of this work are available from the corresponding authors upon reasonable request.

Received: 25 May 2021; Accepted: 26 August 2021;

Published online: 14 September 2021

\section{References}

1. Forrest, S. R. The path to ubiquitous and low-cost organic electronic appliances on plastic. Nature 428, 911-918 (2004).

2. Forrest, S. R. Waiting for Act 2: what lies beyond organic light-emitting diode (OLED) displays for organic electronics? Nanophotonics 10, 31-40 (2021).

3. Riede, M., Spoltore, D. \& Leo, K. Organic solar cells-the path to commercial success. Adv. Energy Mater. 11, 2002653 (2021).
4. Karki, A., Gillett, A. J., Friend, R. H. \& Nguyen, T.-Q. The path to $20 \%$ power conversion efficiencies in nonfullerene acceptor organic solar cells. Adv. Energy Mater. 11, 2003441 (2021).

5. Chang, S.-Y., Cheng, P., Li, G. \& Yang, Y. Transparent polymer photovoltaics for solar energy harvesting and beyond. Joule 2, 1039-1054 (2018).

6. Sheriff, H. K. M., Li, Y., Qu, B. \& Forrest, S. R. Aperiodic optical coatings for neutral-color semi-transparent organic photovoltaics. Appl. Phys. Lett. 118, 033302 (2021)

7. Ravishankar, E. et al. Achieving net zero energy greenhouses by integrating semitransparent organic solar cells. Joule 4, 490-506 (2020).

8. Lin, Y. et al. An electron acceptor challenging fullerenes for efficient polymer solar cells. Adv. Mater. 27, 1170-1174 (2015).

9. Yuan, J. et al. Single-junction organic solar cell with over $15 \%$ efficiency using fused-ring acceptor with electron-deficient core. Joule 3, 1140-1151 (2019).

10. Zhang, M. et al. Single-layered organic photovoltaics with double cascading charge transport pathways: $18 \%$ efficiencies. Nat. Commun. 12, 309 (2021).

11. Liu, Q. et al. 18\% Efficiency organic solar cells. Sci. Bull. 65, 272-275 (2020)

12. Li, Y. et al. Color-neutral, semitransparent organic photovoltaics for power window applications. Proc. Natl Acad. Sci. USA 117, 21147-21154 (2020).

13. Holliday, S. et al. A rhodanine flanked nonfullerene acceptor for solutionprocessed organic photovoltaics. J. Am. Chem. Soc. 137, 898-904 (2015).

14. Burlingame, Q., Ball, M. \& Loo, Y.-L. It's time to focus on organic solar cell stability. Nat. Energy 5, 947-949 (2020).

15. Jiang, Z. et al. Highly efficient organic photovoltaics with enhanced stability through the formation of doping-induced stable interfaces. Proc. Natl Acad. Sci. USA 117, 6391-6397 (2020).

16. Du, X. et al. Efficient polymer solar cells based on non-fullerene acceptors with potential device lifetime approaching 10 years. Joule 3, 215-226 (2019).

17. Wang, Y. et al. Recent progress and challenges toward highly stable nonfullerene acceptor-based organic solar cells. Adv. Energy Mater. 11, 2003002 (2021)

18. Baran, D. et al. Reducing the efficiency-stability-cost gap of organic photovoltaics with highly efficient and stable small molecule acceptor ternary solar cells. Nat. Mater. 16, 363-369 (2017).

19. $\mathrm{Xu}, \mathrm{X}$. et al. Interface-enhanced organic solar cells with extrapolated T80 lifetimes of over 20 years. Sci. Bull. 65, 208-216 (2020).

20. Gasparini, N. et al. Burn-in free nonfullerene-based organic solar cells. Adv. Energy Mater. 7, 1700770 (2017).

21. Peters, C. H. et al. The mechanism of burn-in loss in a high efficiency polymer solar cell. Adv. Mater. 24, 663-668 (2012).

22. Rivaton, A. et al. Photostability of organic materials used in polymer solar cells. Polym. Int. 63, 1335-1345 (2014).

23. Ghasemi, M. et al. Delineation of thermodynamic and kinetic factors that control stability in non-fullerene organic solar cells. Joule 3, 1328-1348 (2019).

24. Burlingame, Q. et al. Intrinsically stable organic solar cells under highintensity illumination. Nature 573, 394-397 (2019).

25. Li, Y. et al. High efficiency near-infrared and semitransparent non-fullerene acceptor organic photovoltaic cells. J. Am. Chem. Soc. 139, 17114-17119 (2017).

26. Lee, B., Lahann, L., Li, Y. \& Forrest, S. R. Cost estimates of production scale semitransparent organic photovoltaic modules for building integrated photovoltaics. Sustain. Energy Fuels 4, 5765-5772 (2020).

27. Reese, M. O. et al. Consensus stability testing protocols for organic photovoltaic materials and devices. Sol. Energy Mater. Sol. Cells 95, 1253-1267 (2011).

28. Forrest, S. R. Organic Electronics: Foundations to Applications (Oxford University Press, 2020).

29. Ye, L. et al. Quantitative relations between interaction parameter, miscibility and function in organic solar cells. Nat. Mater. 17, 253-260 (2018).

30. Park, S. \& Son, H. J. Intrinsic photo-degradation and mechanism of polymer solar cells: the crucial role of non-fullerene acceptors. J. Mater. Chem. A 7, 25830-25837 (2019)

31. Jiang, Y. et al. Photocatalytic effect of $\mathrm{ZnO}$ on the stability of nonfullerene acceptors and its mitigation by $\mathrm{SnO}_{2}$ for nonfullerene organic solar cells. Mater. Horiz. 6, 1438-1443 (2019).

32. Cao, B., Cai, W. \& Zeng, H. Temperature-dependent shifts of three emission bands for $\mathrm{ZnO}$ nanoneedle arrays. Appl. Phys. Lett. 88, 161101 (2006).

33. Classen, A. et al. Revealing hidden UV instabilities in organic solar cells by correlating device and material stability. Adv. Energy Mater. 9, 1902124 (2019).

34. Peters, C. H. et al. High efficiency polymer solar cells with long operating lifetimes. Adv. Energy Mater. 1, 491-494 (2011).

35. White, R. T., Thibau, E. S. \& Lu, Z.-H. Interface structure of $\mathrm{MoO} 3$ on organic semiconductors. Sci. Rep. 6, 21109 (2016).

36. Yan, L. et al. The interfacial degradation mechanism of polymer:fullerene bisadduct solar cells and their stability improvement. Mater. Adv. 1, 1307-1317 (2020).

37. Xie, F. et al. Low-temperature solution-processed hydrogen molybdenum and vanadium bronzes for an efficient hole-transport layer in organic electronics. Adv. Mater. 25, 2051-2055 (2013). 
38. Huang, Y. et al. Nitrogen-doped porous molybdenum carbide and phosphide hybrids on a carbon matrix as highly effective electrocatalysts for the hydrogen evolution reaction. Adv. Energy Mater. 8, 1701601 (2018).

39. Kan, D., Huang, X., Li, Y. \& Forrest, S. R. Photogeneration and the bulk quantum efficiency of organic photovoltaics. Energy Environ. Sci. 14, 1584-1593 (2021).

40. Li, Y. et al. A near-infrared non-fullerene electron acceptor for high performance polymer solar cells. Energy Environ. Sci. 10, 1610-1620 (2017).

41. Giebink, N. C., Wiederrecht, G. P., Wasielewski, M. R. \& Forrest, S. R. Thermodynamic efficiency limit of excitonic solar cells. Phys. Rev. B 83, 195326 (2011).

42. Habte, A., Sengupta, M. \& Lopez, A. Evaluation of the National Solar Radiation Database (NSRDB Version 2): 1998-2015. NREL/TP-5D00-67722 (2017).

43. Liu, H. et al. Boosting organic-metal oxide heterojunction via conjugated small molecules for efficient and stable nonfullerene polymer solar cells. $A d v$. Energy Mater. 9, 1900887 (2019).

44. Li, Y. et al. Enhanced light utilization in semitransparent organic photovoltaics using an optical outcoupling architecture. Adv. Mater. 31, 1903173 (2019).

45. Wang, N., Tong, X., Burlingame, Q., Yu, J. \& Forrest, S. R. Photodegradation of small-molecule organic photovoltaics. Sol. Energy Mater. Sol. Cells 125 170-175 (2014).

46. Burlingame, Q., Zanotti, G., Ciammaruchi, L., Katz, E. A. \& Forrest, S. R Outdoor operation of small-molecule organic photovoltaics. Org. Electron. 41, 274-279 (2017).

47. Hexemer, A. et al. A SAXS/WAXS/GISAXS beamline with multilayer monochromator. J. Phys. Conf. Ser. 247, 012007 (2010).

48. Gann, E. et al. Soft x-ray scattering facility at the Advanced Light Source with real-time data processing and analysis. Rev. Sci. Instrum. 83, 045110 (2012).

\section{Acknowledgements}

The work of Y.L., X.H. and H.S. acknowledge the support of the U.S. Department of Energy's Office of Energy Efficiency and Renewable Energy (EERE) under Solar Energy Technologies Office (SETO) Agreement Number DE-EE0008561. This report was prepared as an account of work sponsored by an agency of the United States Government. Neither the United States Government nor any agency thereof, nor any of their employees, makes any warranty, express or implied, or assumes any legal liability or responsibility for the accuracy, completeness, or usefulness of any information, apparatus, product, or process disclosed, or represents that its use would not infringe privately owned rights. Reference herein to any specific commercial product, process, or service by trade name, trademark, manufacturer, or otherwise does not necessarily constitute or imply its endorsement, recommendation, or favoring by the United States Government or any agency thereof. The views and opinions of authors expressed herein do not necessarily state or reflect those of the United States Government or any agency thereof. Also, K.D. was supported by the Department of the Navy, Office of Naval Research under award no. N00014-17-1-2211, and SRF by Universal Display Corp. L.Y. and H.A. were supported by the Office of Naval Research grant (No. N000141712204) for X-ray data acquisition. L.Y. also thanks the National Natural Science Foundation of China (No. 52073207) and the Peiyang scholar program of Tianjin University for support. X-ray data were acquired at beamlines 7.3.3 and 11.0.1.2 at the Advanced Light Source (ALS) in Lawrence Berkeley National Lab, which is supported by the U.S. Department of Energy
(DE-AC02-05CH11231). Beamline support at ALS by C. Zhu and C. Wang is appreciated. C.L. and H.L. acknowledge the support from the National Key Research and Development Program of China (No. 2019YFA0705900). We also thank Dr. Dejiu Fan and Mr. Boning Qu for helpful discussions, Dr. Yunpeng Qin for X-ray data acquisition and Mr. Cong Zhao for XPS data analysis, and Michigan Center for Materials Characterization (NSF grant \#DMR-0420785).

\section{Author contributions}

Y.L., X.H. and S.R.F. designed the experiments. Y.L. synthesized materials. Y.L. and X.H fabricated all the solar cell samples, conducted the measurements, and performed data analysis. K.D. conducted the transmission electron microscopy measurements and assisted with manuscript preparation. H.S. fabricated UV-filter and performed data analysis. L.Y. and H.A. performed GIWAXS and R-SoXS measurements. C.L. and H.L. developed IC-SAM. S.R.F. supervised the project and analyzed the data. The manuscript was written by Y.L. and S.R.F. and edited by all co-authors.

\section{Competing interests}

One of the authors (S.R.F.) has an ownership interest in one of the sponsors of this research (Universal Display Corp.). This apparent conflict is under management by the University of Michigan's Office of Research. The remaining authors declare no completing interests.

\section{Additional information}

Supplementary information The online version contains supplementary material available at https://doi.org/10.1038/s41467-021-25718-w.

Correspondence and requests for materials should be addressed to Stephen R. Forrest.

Peer review information Nature Communications thanks the anonymous reviewer(s) for their contribution to the peer review of this work.

Reprints and permission information is available at http://www.nature.com/reprints

Publisher's note Springer Nature remains neutral with regard to jurisdictional claims in published maps and institutional affiliations.

Open Access This article is licensed under a Creative Commons Attribution 4.0 International License, which permits use, sharing, adaptation, distribution and reproduction in any medium or format, as long as you give appropriate credit to the original author(s) and the source, provide a link to the Creative Commons license, and indicate if changes were made. The images or other third party material in this article are included in the article's Creative Commons license, unless indicated otherwise in a credit line to the material. If material is not included in the article's Creative Commons license and your intended use is not permitted by statutory regulation or exceeds the permitted use, you will need to obtain permission directly from the copyright holder. To view a copy of this license, visit http://creativecommons.org/ licenses/by/4.0/.

(C) The Author(s) 2021 